\title{
Three loops renormalization constants in Numerical Stochastic Perturbation Theory
}

\section{Masayasu Hasegawa*}

Università di Parma and INFN, Dipartimento di Fisica, Via G.P.Usberti 7/A, 43100 Parma Italy

E-mail: masayasu.hasegawa@fis.unipr.it

\section{Michele Brambilla}

Università di Parma and INFN, Dipartimento di Fisica, Via G.P.Usberti 7/A, 43100 Parma Italy

E-mail: michele.brambillaefis.unipr.it

\section{Francesco Di Renzo}

Università di Parma and INFN, Dipartimento di Fisica, Via G.P.Usberti 7/A, 43100 Parma Italy

E-mail: francesco.direnzodfis.unipr.it

\begin{abstract}
We present three loops renormalization constants for Wilson fermion bilinears (vector, scalar, axial, pseudoscalar currents). Two gluonic regularizations are considered: tree level Symanzik improved action (with $N_{f}=2$ ) and Iwasaki action (with $N_{f}=4$ ). Both cases are amenable for comparisons with non-perturbative results.

We discuss the issue of taming both finite lattice spacing and finite volume artifacts. As a byproduct, we comment on two loops matching of lattice and continuum couplings.
\end{abstract}

The 30 International Symposium on Lattice Field Theory - Lattice 2012,

June 24-29, 2012

Cairns, Australia

${ }^{*}$ Speaker. 


\section{Introduction}

A few years ago the Parma group embarked on an ambitious program: three loops computation of Lattice QCD renormalization constants by means of Numerical Stochastic Perturbation Theory (NSPT) [1]. Results were first presented for finite quantities (i.e. the Zs for vector and axial currents, finite ratios of scalar and pseudoscalar currents) [2]: results could be obtained in the continuum limit, but quite large finite volume effects prevented at that time the reliable computation of logarithmical divergent renormalization constants (e.g. those of the scalar and pseudoscalar currents). A consistent scheme was later introduced [3] to compute finite volume corrections and to allow a careful extraction of the infinite volume limit.

We addressed three loops computations for two schemes which can be relevant for comparison with non-perturbative determinations of the same quantities. The schemes are defined by Wilson fermions and Tree Level Symanzik (TLS) or Iwasaki gauge actions. The number of flavors is $n_{f}=2$ (TLS) an $n_{f}=4$ (Iwasaki) respectively. We notice that, since we work in the massless RI'-MOM scheme, results for (plain) Wilson fermions also hold in a Twisted Mass scheme.

In the following we recall a few issues of our approach: while for the NSPT method we refer the reader to the literature [1], we point out the basic points that are relevant for the computation at hand. We then proceed to the results (still preliminary), which we present here for the first time both for TLS and for Iwasaki actions.

\section{Three loops NSPT computations for RI'-MOM}

The scheme we work in is the masseless RI'-MOM, which is defined by the renormalization conditions

$$
\left.Z_{O_{\Gamma}}(\mu, g) Z_{q}^{-1}(\mu, g) O_{\Gamma}(p)\right|_{p^{2}=\mu^{2}}=1 .
$$

The $O_{\Gamma}(p)$ are defined by projecting onto the three level structure

$$
O_{\Gamma}(p)=\operatorname{Tr}\left(\hat{P}_{O_{\Gamma}} \Gamma_{\Gamma}(p)\right)
$$

the amputated $\Gamma$ functions which come from Landau gauge computation of quark bilinears bracketted in fixed momentum states $(S(p)$ is the quark propagator)

$$
\Gamma_{\Gamma}(p)=S^{-1}(p) G_{\Gamma}(p) S^{-1}(p) \quad G_{\Gamma}(p)=\int d x\langle p|\bar{\psi}(x) \Gamma \psi(x)| p\rangle .
$$

The scheme has been extensively used in Lattice QCD: it is peraphs the most popular one for the non-perturbative computation of renormalization constants. Since there is no conceptual obstacle for a perturbative determination of either finite or logarithically divergent $Z \mathrm{~s}$, a high loops computation opens the way to a valuable cross-check of non-perturbative results. In perturbation theory, in the continuum limit $a \rightarrow 0$ the generic $Z$ reads

$$
Z=1+\sum_{n>0} d_{n}(L) \alpha(\mu)^{n} \quad d_{n}(L)=\sum_{i=0}^{n} d_{n}^{(i)} L^{i} \quad L=\log (\mu a)
$$


where the lattice cutoff $(a)$ is in place and the expansion is in a renormalized coupling. For finite quantities (e.g. vector and axial currents) $d_{n}^{(i)}=0$ for $i>0$. To compute the Zs in NSPT, a main step will be to eventually obtain expansions in the bare coupling $\alpha_{0}$

$$
Z=1+\sum_{n>0} c_{n}(L) \alpha_{0}^{n} .
$$

Such epressions can be directly read from the NSPT simulations, provided one can properly disentangle the finite lattice spacing artifacts. We now proceed to comment on the reasons that make the NSPT computations at hand effective.

- An ovious limitation of a perturbative computation is of course its finite order. In diagramatic perturbation theory (which is very cumbersone in the lattice scheme) two loops computations are in practice the maximum one can achieve. While NSPT can in general address very high orders, one could be worried this can not hold in the computation of Eq. (2.3), since the latter involves logarithms whose determination would require an irrealistic numerical precision. This is actually not the case, since three loops results can be taken for free from the continuum computation of RI'-MOM anomalous dimensions [4]. By differentiating Eq. (2.2) with respect to $\log (\mu a)$ one obtains the expression for the anomalous dimension

$$
\gamma=\frac{1}{2} \frac{d}{d L} \log Z
$$

Demanding that the resulting expression matches the three loops expansion which is known from [4]

$$
\gamma=\sum_{n>0} \gamma_{n} \alpha(L)^{n}
$$

one can read the expressions of all the $d_{n}^{(i)}$ (all the logarithms have to cancel out). The $c_{n}$ of Eq. (2.3) can finally be obtained by re-expressing the expansion in Eq. (2.2) as an expansion in the bare coupling $\alpha_{0}$. The $c_{n}$ turn out to be a function of the $\gamma_{n}$ and of the coefficients of the matching of the continuum and the lattice scheme couplings.

- In order to profit from the previous matchings, a two loops matching of continuum and lattice couplings has to be obtained (only one loop result is known from the literature [5]). We have obtained this by computing the matching to a third scheme, namely the potential scheme defining $\alpha_{V}$

$$
V(R)=2 \delta m-C_{F} \frac{\alpha_{V}(R)}{R} .
$$

By computing convenient Wilson loops (to be definite, Creuts ratios) $\alpha_{V}$ is obtained as a series in the lattice schemes (TLS or Iwasaki) we work in. Since the matching of $\alpha_{V}$ to the continuum coupling we are interested in is known, this immediately leads to the matching we need. For the details of this approach we refer the reader to [6]. We plan to improve the precision of these matching computations by changing the intermediate scheme to take into account the Schroedinger Functional scheme, for which NSPT results are on their way.

- Since the scheme we adhere to is a massless one, one needs to compute everything at zero quark mass. While in the non-perturbative analogue of our computations this requires to go 
through a chiral extrapolation, there is no extrapolation at hand in our case. We recall the definition of the critical mass, i.e. the addittive quark mass renormalization which the Wilson fermions regularization inherits from the breaking of chiral symmetry:

$$
\begin{gathered}
a \Gamma_{2}\left(\hat{p}, \hat{m}_{c r}, \beta^{-1}\right)=a S\left(\hat{p}, \hat{m}_{c r}, \beta^{-1}\right)^{-1} \\
=i \hat{p}+\hat{m}_{W}(\hat{p})-\hat{\Sigma}\left(\hat{p}, \hat{m}_{c r}, \beta^{-1}\right) \\
\hat{\Sigma}\left(\hat{p}, \hat{m}_{c r}, \beta^{-1}\right)=\hat{\Sigma}_{c}\left(\hat{p}, \hat{m}_{c r}, \beta^{-1}\right)+\hat{\Sigma}_{V}\left(\hat{p}, \hat{m}_{c r}, \beta^{-1}\right)+\hat{\Sigma}_{o}\left(\hat{p}, \hat{m}_{c r}, \beta^{-1}\right) \\
\hat{\Sigma}\left(0, \hat{m}_{c r}, \beta^{-1}\right)=\hat{\Sigma}_{c}\left(0, \hat{m}_{c r}, \beta^{-1}\right)=\hat{m}_{c r}
\end{gathered}
$$

Since two loops results are available [7], we can insert this expansion of the critical mass and thus enforce the massless limit in our computations at three loops level. As a byproduct, on top of this we obtained the three loops critical mass.

- Once we know the logarithms which are present in Eq. (2.3), these can be subtracted by the quantities one numerically computes (i.e. the $O_{\Gamma}$ ). As a result, the only remaining dependence on $p a$ is an irrelevant one. This dependence can be fitted by keeping in mind that it must comply to the relevant (H4) symmetry group, e.g. for the quark self-energy $(\hat{p}=p a)$

$$
\hat{\Sigma}\left(\hat{p}, \hat{m}_{c r}, \beta^{-1}\right)=\hat{\Sigma}_{c}\left(\hat{p}, \hat{m}_{c r}, \beta^{-1}\right)+\hat{\Sigma}_{V}\left(\hat{p}, \hat{m}_{c r}, \beta^{-1}\right)+\hat{\Sigma}_{o}\left(\hat{p}, \hat{m}_{c r}, \beta^{-1}\right)
$$

we have

$$
\hat{\Sigma}_{V}=i \sum_{\mu} \gamma_{\mu} \hat{p}_{\mu}\left(\hat{\Sigma}_{V}^{(0)}+\hat{p}_{\mu}^{2} \hat{\Sigma}_{V}^{(1)}+\hat{p}_{\mu}^{4} \hat{\Sigma}_{V}^{(2)}+\ldots\right)
$$

where for example

$$
\hat{\Sigma}_{V}^{(n)}=\alpha_{1}^{(n)} 1+\alpha_{2}^{(n)} \sum_{v} \hat{p}_{v}^{2}+\alpha_{3}^{(n)} \sum_{v} \hat{p}_{v}^{4} / p^{2}+\mathscr{O}\left(a^{4}\right) .
$$

The only term surviving the $a \rightarrow 0$ limit is $\alpha_{1}^{(0)}$, which is the only one we are interested in. Notice that the example of the quark self-energy is trivial at first loop in Landau gauge (one loop anomalous dimension of the quark field being zero); the notation is however valid at any order.

- The last issue we have to deal with are finite size corrections, which are unavoidable since any numerical computation can only be performed on a finite lattice. On dimensional grounds we expect (take once again $\left.\Sigma^{(n)}\right) p L$ effects

$$
\begin{aligned}
\hat{\Sigma}_{V}^{(n)}(\hat{p}, p L) & =\hat{\Sigma}_{V}^{(n)}(\hat{p}, \infty)+\left(\hat{\Sigma}_{V}^{(n)}(\hat{p}, p L)-\hat{\Sigma}_{V}^{(n)}(\hat{p}, \infty)\right) \\
& =\hat{\Sigma}_{V}^{(n)}(\hat{p}, \infty)+\Delta \hat{\Sigma}_{V}^{(n)}(\hat{p}, p L)
\end{aligned}
$$

so that a better expansion to fit is

$$
\begin{aligned}
\hat{\Sigma}_{V}^{(n)}(\hat{p}, p L)= & \alpha_{1}^{(n)} 1+\alpha_{2}^{(n)} \sum_{v} \hat{p}_{v}^{2}+\alpha_{3}^{(n)} \sum_{v} \hat{p}_{v}^{4} / p^{2}+ \\
& +\Delta \hat{\Sigma}_{V}^{(n)}(\hat{p}, p L)+\ldots
\end{aligned}
$$


The key observation is that in first approximation

$$
\Delta \hat{\Sigma}_{V}^{(n)}(\hat{p}, p L) \sim \Delta \hat{\Sigma}_{V}^{(n)}(p L)
$$

But $p_{\mu} L=\frac{2 \pi n_{\mu}}{L} L=2 \pi n_{\mu}$, i.e. we expect the same correction on different lattice sizes for the same $\left\{n_{1}, n_{2}, n_{3}, n_{4}\right\}$. This enable us to fit only a few extra parameters[3].

\section{Results}

\subsection{Critical mass}

As we said, as a byproduct of our computations we obtained the three loops coefficients of the expansion of the critical mass. Results are presented in Tab. (1) and in Tab. (2), referring to $n_{f}=2$ TLS and $n_{f}=4$ Iwasaki respectively. Here and in the following results are presented as expansion in $\beta^{-1}$.

\begin{tabular}{l|l}
\hline$m_{c r}^{(1)}$ & 1.3209 (analytic) \\
\hline$m_{c r}^{(2)}$ & 0.1911 (analytic) \\
\hline$m_{c r}^{(3)}$ & $3.94(4)$ \\
\hline
\end{tabular}

Table 1: Critical mass for $n_{f}=2$ Symanzik

\begin{tabular}{l|l}
\hline$m_{c r}^{(1)}$ & 2.0489 (analytic) \\
\hline$m_{c r}^{(2)}$ & 1.9836 (analytic) \\
\hline$m_{c r}^{(3)}$ & $0.77(2)$ \\
\hline
\end{tabular}

Table 2: Critical mass for $n_{f}=4$ Iwasaki

One does not expect that these expansions can be that meaningful for a power-divergent quantity. Still, it will be interesting to check the outcome of three loops summations in different couplings, a job we postpone to the final publication of results.

\subsection{Currents renormalization coefficients: Tree Level Symanzik}

In Tab. (3) and Tab. (4) we present our results for one, two and three loops of the renormalization constants scalar, pseudoscalr, vector and axial current for $n_{f}=2$ Tree Level Symanzik gauge action (expansions are in $\beta^{-1}$ ). We explicitely contrast our one loop results with analytical ones.

\begin{tabular}{c|l}
\hline Analytic $Z_{S}^{(1)}$ & -0.6893 \\
\hline$Z_{S}^{(1)}$ & $-0.683(5)$ \\
$Z_{S}^{(2)}$ & $-0.789(13)$ \\
$Z_{S}^{(3)}$ & $-1.9(1)$ \\
\hline Analytic $Z_{P}^{(1)}$ & -1.1010 \\
\hline$Z_{P}^{(1)}$ & $-1.098(2)$ \\
$Z_{P}^{(2)}$ & $-1.18(2)$ \\
$Z_{P}^{(3)}$ & $-3.1(2)$ \\
\hline
\end{tabular}

Table 3: Renormalization constants $Z_{S}$ and $Z_{P}$ for $n_{f}=2$ Symanzik gauge action

\begin{tabular}{c|l}
\hline Analytic $Z_{V}^{(1)}$ & -0.8411 \\
\hline$Z_{V}^{(1)}$ & $-0.834(6)$ \\
$Z_{V}^{(2)}$ & $-0.896(12)$ \\
$Z_{V}^{(3)}$ & $-1.88(3)$ \\
\hline Analytic $Z_{A}^{(1)}$ & -0.6352 \\
\hline$Z_{A}^{(1)}$ & $-0.644(26)$ \\
$Z_{A}^{(2)}$ & $-0.618(9)$ \\
$Z_{A}^{(3)}$ & $-1.21(3)$ \\
\hline
\end{tabular}

Table 4: Renormalization constants $Z_{V}$ and $Z_{A}$ for $n_{f}=2$ Symanzik gauge action 


\subsection{Currents renormalization coefficients: Iwasaki}

In Tab. (5) and Tab. (6) we present our results for one, two and three loops of the renormalization constants scalar, pseudoscalr, vector and axial current for $n_{f}=4$ Iwasaki gauge action (expansions are in $\beta^{-1}$ ).

\begin{tabular}{c|l}
\hline Analytic $Z_{S}^{(1)}$ & -0.4488 \\
\hline$Z_{S}^{(1)}$ & $-0.435(9)$ \\
$Z_{S}^{(2)}$ & $-0.16(2)$ \\
$Z_{S}^{(3)}$ & $-0.82(5)$ \\
\hline Analytic $Z_{P}^{(1)}$ & -0.7433 \\
\hline$Z_{P}^{(1)}$ & $-0.735(3)$ \\
$Z_{P}^{(2)}$ & $-0.202(8)$ \\
$Z_{P}^{(3)}$ & $-1.01(9)$ \\
\hline
\end{tabular}

Table 5: Renormalization constants $Z_{S}$ and $Z_{P}$ for $n_{f}=4$ Iwasaki gauge action

\begin{tabular}{c|l}
\hline Analytic $Z_{V}^{(1)}$ & -0.5623 \\
\hline$Z_{V}^{(1)}$ & $-0.553(6)$ \\
$Z_{V}^{(2)}$ & $-0.073(9)$ \\
$Z_{V}^{(3)}$ & $-0.37(4)$ \\
\hline Analytic $Z_{A}^{(1)}$ & -0.4150 \\
\hline$Z_{A}^{(1)}$ & $-0.410(4)$ \\
$Z_{A}^{(2)}$ & $-0.055(9)$ \\
$Z_{A}^{(3)}$ & $-0.079(16)$ \\
\hline
\end{tabular}

Table 6: Renormalization constants $Z_{V}$ and $Z_{A}$ for $n_{f}=4$ Iwasaki gauge action

\section{Conclusions and Future}

Three loop computations for renormalization constants are feasible, with good control on systematics. A few final comments are in order:

- Perturbative corrections to leading order appear to be small for Iwasaki action. One should in any case keep in mind that the values of $\beta$ corresponding to comparable values of the lattice spacing are roughly different by a factor of two with respect to TLS action.

- To make a meaningful comparison with non-perturbative results [8], we are going to assess summations of the expansions in different couplings: we contrast this with what is usually referred to as boosted perturbation at one loop level of accuracy, not to be regarded as really stable. This job will be dealt with in the final publication.

- Results are still preliminary. A few technical details to improve our fitting procedure of the irrelevant contributions were still under development at the time of the conference.

\section{Acknowledgements}

We ackowledge computer time made available on Tramontana and Aurora in Italy, RCNP and CMC in Japan. This work is supported by I.N.F.N. under the research project MI11, by the Research Executive Agency (REA) of the European Union under Grant Agreement number PITNGA-2009-238353 (ITN STRONGnet) and by MIUR contract PRIN2009 (20093BMNPR_004). 


\section{References}

[1] F. Di Renzo and L. Scorzato, Numerical Stochastic Perturbation Theory for full QCD, JHEP 0410 (2004) 073 [arXiv:hep-lat/0410010].

[2] F. Di Renzo, V. Miccio, L. Scorzato and C. Torrero, High-loop perturbative renormalization constants for Lattice QCD. I. Finite constants for Wilson quark currents., Eur. Phys. J. C51 (2007) 645-657 [arXiv:hep-lat/0611013].

[3] F. Di Renzo, E. -M. Ilgenfritz, H. Perlt, A. Schiller and C. Torrero, Two-point functions of quenched lattice QCD in Numerical Stochastic Perturbation Theory. (I) The ghost propagator in Landau gauge, Nucl. Phys. B831 (2010) 262-284 [arXiv:0912.4152 [hep-lat]].

F. Di Renzo, E. -M. Ilgenfritz, H. Perlt, A. Schiller and C. Torrero, Two-point functions of quenched lattice QCD in Numerical Stochastic Perturbation Theory. (II) The Gluon propagator in Landau gauge, Nucl. Phys. B842, 122-139 (2011) [arXiv:1008.2617 [hep-lat]].

[4] J. A. Gracey, Three loop anomalous dimension of nonsinglet quark currents in the RI-prime scheme, Nucl. Phys. B 662, 247 (2003) [arXiv:hep-ph/0304113].

[5] S. Aoki, K. i. Nagai, Y. Taniguchi and A. Ukawa, Perturbative renormalization factors of bilinear quark operators for improved gluon and quark actions in lattice QCD, Phys. Rev. D 58, 074505 (1998) [arXiv:hep-lat/9802034].

[6] M. Brambilla and F. Di Renzo, Matching the lattice coupling to the continuum for the tree level Symanzik improved gauge action, Pos (Lattice2010) 222.

[7] A. Skouroupathis, M. Constantinou, H. Panagopoulos, Two-loop additive mass renormalization with clover fermions and Symanzik improved gluons, Phys. Rev. D77 (2008) 014513 [arXiv:0801.3146 [hep-lat]].

[8] M. Constantinou et al. [ETM Collaboration], Non-perturbative renormalization of quark bilinear operators with $N_{f}=2$ (tmQCD) Wilson fermions and the tree-level improved gauge action, JHEP 1008, 068 (2010) [arXiv:1004.1115 [hep-lat]]. B. Blossier et al. [ETM Collaboration], Renormalisation constants of quark bilinears in lattice QCD with four dynamical Wilson quarks, PoS LATTICE 2011, 233 (2011) [arXiv:1112.1540 [hep-lat]]. 